\title{
UNIFORM APPROXIMATION OF MUCKENHOUPT WEIGHTS ON FRACTALS BY SIMPLE FUNCTIONS
}

\author{
MARILINA CARENA AND MARISA TOSCHI
}

\begin{abstract}
Given an $A_{p}$-Muckenhoupt weight on a fractal obtained as the attractor of an iterated function system, we construct a sequence of approximating weights, which are simple functions belonging uniformly to the $A_{p}$ class on the approximating spaces.
\end{abstract}

\section{INTRODUCTION}

Let us start by describing our general framework. Let $(X, d)$ be a compact metric space with diameter 1, i.e. $\sup _{x, y \in X} d(x, y)=1$. Let $\mu$ be a Borel probability on $X$ such that there exist constants $K_{1}, K_{2}$, and $\gamma>0$ for which the inequalities

$$
K_{1} r^{\gamma} \leq \mu(B(x, r)) \leq K_{2} r^{\gamma}
$$

hold for every $x \in X$ and $r \in(0,1]$. Sometimes this property is called Ahlfors condition or is described by saying that $(X, d, \mu)$ is an Ahlfors space of dimension $\gamma$. It is easy to see that such space $(X, d, \mu)$ is a space of homogeneous type. This means that there exists a constant $A \geq 1$ such that $0<\mu(B(x, 2 r)) \leq A \mu(B(x, r))<\infty$ for every $x \in X$ and every $r>0$.

Let $\Phi$ be a finite set of contractive similitudes $\Phi=\left\{\phi_{i}: X \rightarrow X, i=1,2, \ldots, H\right\}$ with the same contraction rate. Precisely, each $\phi_{i}$ satisfies

$$
d\left(\phi_{i}(x), \phi_{i}(y)\right)=\beta d(x, y)
$$

for every $x, y \in X$ and some constant $0<\beta<1$. The set $\Phi$ is called an iterated function system (IFS). We shall assume that $\Phi$ satisfies the open set condition (OSC). This means that there exists a non-empty open set $U \subset X$ such that

$$
\bigcup_{i=1}^{H} \phi_{i}(U) \subseteq U,
$$

and $\phi_{i}(U) \cap \phi_{j}(U)=\emptyset$ if $i \neq j$. When $U$ satisfies these properties we say that $U$ is a set for the $O S C$ for $\Phi$.

2020 Mathematics Subject Classification. 28A80, 42B25.

Key words and phrases. Muckenhoupt weights; iterated function systems; weak convergence. The authors were supported by CONICET, CAI+D (UNL) and ANPCyT. 
For $n \in \mathbb{N}$, set $\mathfrak{I}^{n}=\{1,2, \ldots, H\}^{n}$. Given $\boldsymbol{i}=\left(i_{1}, i_{2}, \ldots, i_{n}\right) \in \mathfrak{I}^{n}$, we denote by $\phi_{i}^{n}$ the composition $\phi_{i_{n}} \circ \phi_{i_{n-1}} \circ \cdots \circ \phi_{i_{2}} \circ \phi_{i_{1}}$. Then for any subset $E$ of $X$ we have $\phi_{i}^{n}(E)=\left(\phi_{i_{n}} \circ \phi_{i_{n-1}} \circ \cdots \circ \phi_{i_{2}} \circ \phi_{i_{1}}\right)(E)$. Set $X_{i}^{n}=\phi_{i}^{n}(X)$ and $X^{n}=\bigcup_{i \in \mathfrak{I}^{n}} X_{i}^{n}$.

It is well known that the sequence of sets $\left(X^{n}\right)_{n}$ converges in the sense of the Hausdorff distance to a non-empty compact set $X^{\infty}$, which is called the attractor of the system $\Phi$. This set satisfies

$$
X^{\infty}=\bigcup_{i=1}^{H} \phi_{i}\left(X^{\infty}\right)
$$

and it is the only set in $X$ satisfying this property. Moreover, since $\phi_{i}(X) \subseteq X$ for every $i$, we have that $X^{\infty}=\bigcap_{n=1}^{\infty} X^{n}$ (see [6] or [4]), so that the system $\Phi$ defines or represents the set $X^{\infty}$.

We shall also assume that $\Phi$ satisfies the adjacency property. That means that there exists a positive constant $c$ such that the inclusion

$$
B\left(\phi_{i}^{n}(z), r\right) \cap X_{j}^{n} \subseteq B\left(\phi_{j}^{n}(z), c r\right) \cap X_{j}^{n}
$$

holds for every $n \in \mathbb{N}$, every $\boldsymbol{i}, \boldsymbol{j} \in \mathfrak{I}^{n}$, every $r>0$, and every $z \in X$.

As a simple example, the system $\Phi=\{x / 3, x / 3+2 / 3\}$ associated to the middlethird Cantor set satisfies the adjacency property on $X=[0,1]$. Also, some of the classical fractals can be obtained through somehow non-standard IFS's satisfying this property. For example, for the Sierpinski gasket we can slightly modify the usual IFS defined on the triangle $X$ with vertices at $a=(0,0), b=(1 / 2, \sqrt{3} / 2)$, and $c=(1,0)$. If $\rho_{\theta}$ denotes the rotation of $\theta$ radians about the origin of $\mathbb{R}^{2}$ in the positive sense, we have that the IFS given by $\Phi=\left\{\phi_{1}, \phi_{2}, \phi_{3}\right\}$, where

$$
\begin{aligned}
\phi_{1}(x, y) & =\frac{1}{2}(x, y), \\
\phi_{2}(x, y) & =\frac{1}{2}\left(\rho_{4 \pi / 3}(x, y)\right)+\boldsymbol{v}, \\
\phi_{3}(x, y) & =\frac{1}{2}\left(\rho_{2 \pi / 3}(x, y)\right)+\boldsymbol{v},
\end{aligned}
$$

with $\boldsymbol{v}=\left(\frac{3}{4}, \frac{\sqrt{3}}{4}\right)$, satisfies the adjacency property (see [1]), the OSC, and gives rise to the standard Sierpinski triangle. For the Sierpinski carpet, the same is true with $\Phi=\left\{\phi_{i}: 1 \leq i \leq 8\right\}$ given by

$$
\begin{aligned}
\phi_{1}(x, y) & =\frac{1}{3}(x, y), & \phi_{2}(x, y) & =T_{\frac{2}{3}, 0}\left(S_{2}\left(\phi_{1}(x, y)\right)\right), \\
\phi_{3}(x, y) & =T_{\frac{2}{3}, 0}\left(\phi_{1}(x, y)\right), & \phi_{4}(x, y) & =T_{0, \frac{2}{3}}\left(S_{1}\left(\phi_{1}(x, y)\right)\right), \\
\phi_{5}(x, y) & =T_{\frac{2}{3}, \frac{2}{3}}\left(S_{1}\left(\phi_{1}(x, y)\right)\right), & \phi_{6}(x, y) & =T_{0, \frac{2}{3}}\left(\phi_{1}(x, y)\right), \\
\phi_{7}(x, y) & =T_{\frac{2}{3}, \frac{2}{3}}\left(S_{2}\left(\phi_{1}(x, y)\right)\right), & \phi_{8}(x, y) & =T_{\frac{2}{3}, \frac{2}{3}}\left(\phi_{1}(x, y)\right),
\end{aligned}
$$

defined on the unit square $X$ of $\mathbb{R}^{2}$ with vertices $(0,0),(1,0),(1,1)$, and $(0,1)$, where $T_{a, b}(x, y)=(x+a, y+b), S_{1}(x, y)=(x,-y)$, and $S_{2}(x, y)=(-x, y)$. 
Now, we need to define three maximal operators which are related through Theorem 3 of [1]. We will state and use this theorem to prove one of the two main result of this work.

The first of these operators is the Hardy-Littlewood centered maximal function in $(X, d, \mu)$ given by

$$
M h(x)=\sup _{r>0} \frac{1}{\mu(B(x, r))} \int_{B(x, r)}|h(y)| d \mu(y),
$$

for $h \in L_{\mathrm{loc}}^{1}(X, \mu)$.

The second one is a discrete version of the Hardy-Littlewood maximal operator applied to a real function $g$ defined on $\mathfrak{I}^{n}$. Let us fix $x_{0} \in U$ and for $\boldsymbol{i}, \boldsymbol{j} \in \mathfrak{I}^{n}$ define $\tilde{d}(\boldsymbol{i}, \boldsymbol{j})=d\left(\boldsymbol{\phi}_{\boldsymbol{i}}^{n}\left(x_{0}\right), \boldsymbol{\phi}_{\boldsymbol{j}}^{n}\left(x_{0}\right)\right)$. For $n \in \mathbb{N}, \boldsymbol{i} \in \mathfrak{I}^{n}$, and $r>0$, set $\mathcal{B}(\boldsymbol{i}, r)$ to denote the $\tilde{d}$-ball of radius $r$ in $\left(\mathfrak{I}^{n}, \tilde{d}\right)$. More precisely,

$$
\mathcal{B}(\boldsymbol{i}, r)=\left\{\boldsymbol{j} \in \mathfrak{I}^{n}: d\left(\boldsymbol{\phi}_{\boldsymbol{i}}^{n}\left(x_{0}\right), \boldsymbol{\phi}_{\boldsymbol{j}}^{n}\left(x_{0}\right)\right)<r\right\} .
$$

We shall consider the Hardy-Littlewood type maximal function as

$$
\mathfrak{M}_{n} g(\boldsymbol{i})=\sup _{r>0} \frac{1}{\operatorname{card}(\mathcal{B}(\boldsymbol{i}, r))} \sum_{\boldsymbol{j} \in \mathcal{B}(\boldsymbol{i}, r)}|g(\boldsymbol{j})|,
$$

where $\operatorname{card}(E)$ denotes the number of elements of the set $E$. We have to point out that $\tilde{d}$ and hence the $\mathfrak{M}_{n}$ 's depend on $x_{0} \in U$, but we shall fix it from now on.

The last maximal operator is given by

$$
M_{n} h(x)=\sup _{r>0} \frac{1}{\mu^{n}(B(x, r))} \int_{B(x, r)}|h(y)| d \mu^{n}(y),
$$

for $h \in L_{\text {loc }}^{1}\left(X^{n}, \mu^{n}\right)$, where $\mu^{n}$ is the natural uniformly distributed probability measure induced by $\mu$ on $X^{n}$. More precisely,

$$
\mu^{n}(E)=\frac{1}{H^{n}} \sum_{j \in \mathfrak{I}^{n}} \mu\left(\left(\phi_{j}^{n}\right)^{-1}(E)\right)
$$

for $E$ a Borel set in $X^{n}$. In other words, $\mu^{n}=H^{-n} \sum_{j \in \mathfrak{I}^{n}} \mu_{j}^{n}$, with $\mu_{j}^{n}(E)=$ $\mu\left(\left(\phi_{j}^{n}\right)^{-1}(E)\right)$. Notice that $M_{0}=M$ under the standard assumption $X^{0}=X$ and $\mu^{0}=\mu$.

It is well known that the sequence of measures $\left(\mu^{n}\right)_{n}$ converges in the weak star sense to a Borel probability measure $\mu^{\infty}$ supported on the attractor $X^{\infty}$. This measure is called invariant measure or self-affine measure, since it is the unique one satisfying

$$
\mu^{\infty}(A)=\frac{1}{H} \sum_{i=1}^{H} \mu^{\infty}\left(\phi_{i}^{-1}(A)\right)
$$

for every Borel set $A$, and also

$$
\int \varphi(x) d \mu^{\infty}(x)=\frac{1}{H} \sum_{i=1}^{H} \int \varphi\left(\phi_{i}(x)\right) d \mu^{\infty}(x),
$$


for every continuous function $\varphi$ on $X$ (see [6] or [4]). Moreover, the results in [7] show that $\left(X^{\infty}, d, \mu^{\infty}\right)$ is an Ahlfors space of dimension $s=-\log _{\beta} H$.

Let us finally introduce a definition that shall be used in Theorem 2.3 . We shall say that the system $\Phi$ has null overlapping if $\mu^{n}\left(X_{i}^{n} \cap X_{j}^{n}\right)=\mu^{\infty}\left(X_{i}^{n} \cap X_{j}^{n}\right)=0$ for every $n$ and every $\boldsymbol{i}, \boldsymbol{j} \in \mathfrak{I}^{n}, \boldsymbol{i} \neq \boldsymbol{j}$. In other words, $\Phi$ has null overlapping if $\mu^{n}\left(\left\{z: \sum_{\boldsymbol{i} \in \mathfrak{I}^{n}} \mathcal{X}_{X_{\boldsymbol{i}}^{n}}(z)>1\right\}\right)=\mu^{\infty}\left(\left\{z: \sum_{\boldsymbol{i} \in \mathfrak{I}^{n}} \mathcal{X}_{X_{\boldsymbol{i}}^{n}}(z)>1\right\}\right)=0$.

Remark 1.1. This property is not too strong, in the sense that the most of the typical fractals satisfy it. In particular, the examples given above have null overlapping. This property is equivalent to the measures $\mu^{n}$ and $\mu^{\infty}$ being uniformly distributed, in the sense that $\mu^{\infty}\left(X_{j}^{n}\right)=\mu^{n}\left(X_{j}^{n}\right)=H^{-n}$ for every $\boldsymbol{j} \in \mathfrak{I}^{n}$. In fact, taking $A=X_{j}^{n}$ in

$$
\mu^{\infty}(A)=\frac{1}{H} \sum_{i=1}^{H} \mu^{\infty}\left(\phi_{i}^{-1}(A)\right)
$$

we easily obtain that $\mu^{\infty}\left(X_{j}^{n}\right) \geq H^{-n}$. On the other hand, if $\mu^{\infty}\left(X_{j}^{n}\right)>H^{-n}$ for some $\boldsymbol{j} \in \mathfrak{I}^{n}$, from the null overlapping property of the system $\Phi$ we obtain $\mu^{\infty}\left(X^{\infty}\right)>1$. Since $\mu^{\infty}$ is a probability measure on $X^{\infty}$, we have that $\mu^{\infty}\left(X_{i}^{n}\right)=$ $H^{-n}$, and the same holds for $\mu^{n}$.

With the above notation, we can now state the following result, proved in [1].

Theorem 1.2. There exists a geometric constant $C$ such that the inequality

$$
M_{n} f\left(\phi_{i}^{n}(z)\right) \leq C \mathfrak{M}_{n}\left(M\left(f \circ \phi^{n}\right)(z)\right)(\boldsymbol{i})
$$

holds for every $f \in L^{1}\left(X^{n}, \mu^{n}\right), z \in X, \boldsymbol{i} \in \mathfrak{I}^{n}$, and $n \in \mathbb{N}$, where $M\left(f \circ \phi^{n}\right)(z)$ denotes the function $g$ on $\mathfrak{I}^{n}$ defined by $g(\boldsymbol{j})=M\left(f \circ \boldsymbol{\phi}_{\boldsymbol{j}}^{n}\right)(z)$.

Also we shall collect in the next lemma some elementary properties which are proved in [1] and [3].

Lemma 1.3. Let $(X, d, \mu)$ and $\Phi$ as above.

(1) The sequence $\left\{\left(X^{n}, d, \mu^{n}\right): n \in \mathbb{N}\right\}$ is a uniform family of spaces of homogeneous type. In other words, there exists a constant $\tilde{A}$ such that

$$
0<\mu^{n}(B(x, 2 r)) \leq \tilde{A} \mu^{n}(B(x, r))
$$

for every $r>0, x \in X^{n}$, and $n \in \mathbb{N}$.

(2) For each $n \in \mathbb{N}$ we consider the set $\Delta_{n}=\left\{\phi_{j}^{n}\left(x_{0}\right): j \in \mathfrak{I}^{n}\right\}$. Then

(a) for every $n \in \mathbb{N}$ we have that $\Delta_{n}$ is a $\delta \beta^{n}$-disperse set, with $\delta=$ $\operatorname{dist}\left(x_{0}, \partial U\right)$. This means that $d\left(\boldsymbol{\phi}_{\boldsymbol{j}}^{n}\left(x_{0}\right), \boldsymbol{\phi}_{\boldsymbol{i}}^{n}\left(x_{0}\right)\right) \geq \delta \beta^{n}$ for every $\boldsymbol{i} \neq \boldsymbol{j}$ in $\mathfrak{I}^{n}$

(b) $\left\{\left(\Delta_{n}, d\right.\right.$, card $\left.): n \in \mathbb{N}\right\}$ is a sequence of spaces of homogeneous type with a uniform doubling constant $\widehat{A}$.

(3) If $h$ is an integrable real function on $(X, \mu)$ then for each $n \in \mathbb{N}$ and $\boldsymbol{j} \in \mathfrak{I}^{n}$ the function $h \circ \phi_{j}^{n}$ is integrable on $\left(X_{j}^{n}, \mu_{j}^{n}\right)$ and

$$
\int_{X} h \circ \phi_{j}^{n} d \mu=\int_{X_{j}^{n}} h d \mu_{j}^{n} .
$$




\section{MAIN RESUltS}

A weight $w$ is a non-negative function in $L_{\text {loc }}^{1}(X, \mu)$. Given $1<p<\infty$, we shall say that a weight $w$ is an $A_{p}$-Muckenhoupt weight on $(X, \mu)$ if there exists a constant $C$ such that the inequality

$$
\left(\int_{B} w d \mu\right)\left(\int_{B} w^{-\frac{1}{p-1}} d \mu\right)^{p-1} \leq C(\mu(B))^{p}
$$

holds for every $d$-ball $B$ in $X$. We shall also use the notation $w \in A_{p}(X, d, \mu)$, and we shall say that $C$ is a Muckenhoupt constant for $w$. It is well known that if $(X, d, \mu)$ is a space of homogeneous type and $w \in A_{p}(X, d, \mu)$, then $\|M f\|_{L^{p}(d \mu)} \leq$ $C_{p}\|f\|_{L^{p}(d \mu)}$. Actually, a weight $w$ is an $A_{p^{-}}$-Muckenhoupt weight on $(X, \mu)$ if and only if $\|M f\|_{L^{p}(w d \mu)} \leq C_{p, w}\|f\|_{L^{p}(w d \mu)}$ (see [8]). A classical reference for the theory of Muckenhoupt weights in the Euclidean space is [5].

We will say that a sequence $\left(w_{n}\right)$ of weights is uniformly in $A_{p}$, when the $A_{p}$ condition holds with a constant independent of $n$.

We shall use the symbol $\stackrel{*}{\rightarrow}$ to denote the weak star convergence of measures. Also, we shall use the letter $C$ to denote a generic constant, not necessarily the same at each occurrence. The next statement contains the first of the two main results of the paper.

Theorem 2.1. Let $\nu^{n}=\sum_{\boldsymbol{i} \in \mathfrak{I}^{n}} v(\boldsymbol{i}) \mu_{\boldsymbol{i}}^{n}$, with $\mu_{\boldsymbol{i}}^{n}(E)=\mu\left(\left(\phi_{\boldsymbol{i}}^{n}\right)^{-1}(E)\right)$. Then

(1) if $v(\boldsymbol{i}) \in A_{p}\left(\mathfrak{I}^{\mathfrak{n}}, \tilde{d}\right.$, card) uniformly in $n$, then $d \nu^{n}=w_{n} d \mu^{n}$, with $w_{n} \in$ $A_{p}\left(X^{n}, d, \mu^{n}\right)$ uniformly in $n$;

(2) if also we have that $\nu^{n} \stackrel{*}{\rightarrow} \nu$, then $d \nu=w d \mu$, with $w \in A_{p}\left(X^{\infty}, d, \mu^{\infty}\right)$.

Proof. Using Theorem 1.2 the hypothesis (1), and the $L^{p}$ boundedness of $M$ on $(X, d, \mu)$ and $\mathfrak{M}_{n}$ on $\left(\mathfrak{I}^{\mathfrak{n}}, d\right.$, card $)$, we obtain

$$
\begin{aligned}
\int_{X^{n}}\left|M_{n} f\right|^{p} d \nu^{n} & =\sum_{\boldsymbol{i} \in \mathfrak{I}^{n}} \int_{X}\left|M_{n} f\left(\boldsymbol{\phi}_{\boldsymbol{i}}^{n}(z)\right)\right|^{p} v(\boldsymbol{i}) d \mu(z) \\
& \leq C \int_{X} \sum_{\boldsymbol{i} \in \mathfrak{I}^{n}}\left|\mathfrak{M}_{n}\left(M\left(f \circ \boldsymbol{\phi}^{n}\right)(z)\right)(\boldsymbol{i})\right|^{p} v(\boldsymbol{i}) d \mu(z) \\
& \leq C \int_{X} \sum_{\boldsymbol{i} \in \mathfrak{I}^{n}}\left|M\left(f \circ \boldsymbol{\phi}_{\boldsymbol{i}}^{n}\right)(z)\right|^{p} v(\boldsymbol{i}) d \mu(z) \\
& \leq C \sum_{\boldsymbol{i} \in \mathfrak{I}^{n}} \int_{X}\left|\left(f \circ \boldsymbol{\phi}_{\boldsymbol{i}}^{n}\right)(z)\right|^{p} d \mu(z) v(\boldsymbol{i}) \\
& =C \int_{X^{n}}|f|^{p} d \nu^{n} .
\end{aligned}
$$

Then, by [2, Theorem 4] we have that $\nu^{n}$ is absolutely continuous with respect to $\mu^{n}$, and its Radon-Nikodym derivative is an $A_{p}\left(X^{n}, d, \mu^{n}\right)$ weight. Finally, if $\nu^{n} \stackrel{*}{\rightarrow} \nu$, by [2, Theorem 8] we have that $\nu$ is absolutely continuous with respect to $\mu^{\infty}$, and its Radon-Nikodym derivative is an $A_{p}\left(X^{\infty}, d, \mu^{\infty}\right)$ weight. 
In order deal with the second result, we shall state and prove the following auxiliary lemma.

Lemma 2.2. There exist constants $c_{1}$ and $c_{2}$ such that

$$
\bigcup_{\boldsymbol{j} \in \mathcal{B}(\boldsymbol{i}, r)} X_{\boldsymbol{j}}^{n} \subseteq B\left(\phi_{\boldsymbol{i}}^{n}\left(x^{\infty}\right), c_{1} r\right) \subseteq \bigcup_{\boldsymbol{j} \in \mathcal{B}\left(\boldsymbol{i}, c_{2} r\right)} B\left(\phi_{\boldsymbol{j}}^{n}\left(x_{0}\right), \beta^{n}\right),
$$

for every $n \in \mathbb{N}, r \geq \delta \beta^{n}, \boldsymbol{i} \in \mathfrak{I}^{n}$, and $x^{\infty} \in X^{\infty}$. As before, $\delta=\operatorname{dist}\left(x_{0}, \partial U\right)$.

Proof. Let us fix $n \in \mathbb{N}, r \geq \delta \beta^{n}, \boldsymbol{i} \in \mathfrak{I}^{n}$, and $x^{\infty} \in X^{\infty}$. Let $x=\phi_{i}^{n}\left(x^{\infty}\right)$. Fix also $\boldsymbol{j} \in B(\boldsymbol{i}, r)$ and $y \in X_{\boldsymbol{j}}^{n}$. Then we have that $d\left(\boldsymbol{\phi}_{\boldsymbol{j}}^{n}\left(x_{0}\right), \boldsymbol{\phi}_{\boldsymbol{i}}^{n}\left(x_{0}\right)\right)<r$ and that there exists $y_{0} \in X$ such that $y=\phi_{j}^{n}\left(y_{0}\right)$. Then

$$
\begin{aligned}
d(y, x) & \leq d\left(\boldsymbol{\phi}_{\boldsymbol{j}}^{n}\left(y_{0}\right), \boldsymbol{\phi}_{\boldsymbol{j}}^{n}\left(x_{0}\right)\right)+d\left(\boldsymbol{\phi}_{\boldsymbol{j}}^{n}\left(x_{0}\right), \boldsymbol{\phi}_{\boldsymbol{i}}^{n}\left(x_{0}\right)\right)+d\left(\boldsymbol{\phi}_{\boldsymbol{i}}^{n}\left(x_{0}\right), x\right) \\
& \leq \beta^{n} d\left(x_{0}, y_{0}\right)+r+\beta^{n} \leq c_{1} r,
\end{aligned}
$$

where $c_{1}=\frac{2}{\delta}+1$. Therefore $\bigcup_{j \in B} X_{j}^{n} \subseteq B\left(x, c_{1} r\right)$, which proves the first inclusion. In order to prove the second one, $\operatorname{since} \operatorname{diam}\left(X_{j}^{n}\right)=\beta^{n}$, it is enough to estimate the number of sets $X_{j}^{n}$ which intersect $B\left(x, c_{1} r\right)$. In other words, we only have to prove that if $\boldsymbol{j}$ is such that $X_{\boldsymbol{j}}^{n} \cap B\left(x, c_{1} r\right) \neq \emptyset$, then $\boldsymbol{j} \in B\left(\boldsymbol{i}, c_{2} r\right)$ for some $c_{2}$. Indeed, for such $\boldsymbol{j}$ there exists $y \in X_{j}^{n}$ with $d(y, x)<c_{1} r$. Then

$$
\begin{aligned}
\tilde{d}(\boldsymbol{j}, \boldsymbol{i}) & =d\left(\boldsymbol{\phi}_{\boldsymbol{j}}^{n}\left(x_{0}\right), \boldsymbol{\phi}_{\boldsymbol{i}}^{n}\left(x_{0}\right)\right) \\
& \leq d\left(\boldsymbol{\phi}_{\boldsymbol{j}}^{n}\left(x_{0}\right), y\right)+d(y, x)+d\left(x, \boldsymbol{\phi}_{\boldsymbol{i}}^{n}\left(x_{0}\right)\right) \\
& \leq \beta^{n}+c_{1} r+\beta^{n} \leq c_{2} r,
\end{aligned}
$$

where $c_{2}=\frac{4}{\delta}+1$, which completes the proof.

We are now in a position to state and prove the second main result of this work.

Theorem 2.3. Assume that the system $\Phi$ has null overlapping. Given a weight $w \in A_{p}\left(X^{\infty}, d, \mu^{\infty}\right)$, for each natural number $n$ let us define a measure $\nu^{n}$ on $X$ by

$$
\nu^{n}:=\sum_{i \in \mathfrak{I}^{n}} v(\boldsymbol{i}) d \mu_{\boldsymbol{i}}^{n}
$$

where $v(\boldsymbol{i}):=\frac{1}{H^{n} \mu^{\infty}\left(X_{i}^{n}\right)} \int_{X_{i}^{n}} w(y) d \mu^{\infty}(y):=\frac{1}{H^{n}} f_{X_{i}^{n}} w(y) d \mu^{\infty}(y)$. Then $d \nu^{n}=$ $w_{n} d \mu^{n}$, with $w_{n} \in A_{p}\left(X^{n}, \mu^{n}\right)$ uniformly in $n$, and $\nu^{n} \stackrel{*}{\rightarrow} \nu$, where $d \nu:=w d \mu^{\infty}$.

Moreover,

$$
w_{n}(x)=\sum_{i \in \mathfrak{I}^{n}}\left(f_{X_{i}^{n}} w(y) d \mu^{\infty}(y)\right) \mathcal{X}_{X_{i}^{n}}(x),
$$

where $\mathcal{X}_{A}$ denotes the indicator function of the set $A$.

Proof. From (1) in Theorem 2.1 in order to have $d \nu^{n}=w_{n} d \mu^{n}$ with $w_{n}$ belonging to $A_{p}\left(X^{n}, d, \mu^{n}\right)$ uniformly in $n$, it is enough to prove that $v(\boldsymbol{i}) \in A_{p}\left(\mathfrak{I}^{n}, \tilde{d}\right.$, card $)$ 
with constant which does not depend on $n$. Then we shall fix $n, \boldsymbol{i} \in \mathfrak{I}^{n}$, and $r>0$. Let $\mathcal{B}:=\mathcal{B}(\boldsymbol{i}, r)$. We have to prove that

$$
\left(\sum_{\boldsymbol{j} \in \mathcal{B}} v(\boldsymbol{j})\right)\left(\sum_{\boldsymbol{j} \in \mathcal{B}} v(\boldsymbol{j})^{\frac{1}{1-p}}\right)^{p-1} \leq C \operatorname{card}(\mathcal{B})^{p},
$$

for some constant $C$ which does not depend on $\mathcal{B}$ or $n$. Notice that if $r<\delta \beta^{n}$, from $2 \mathrm{a}$ in Lemma 1.3 we have that $\mathcal{B}(\boldsymbol{i}, r)=\boldsymbol{i}$ and the above inequality trivially holds with $C=1$. Then we shall assume that $r \geq \delta \beta^{n}$, and we will show that

$$
\left(\sum_{\boldsymbol{j} \in \mathcal{B}} f_{X_{j}^{n}} w(y) d \mu^{\infty}(y)\right)\left(\sum_{\boldsymbol{j} \in \mathcal{B}}\left(f_{X_{j}^{n}} w(y) d \mu^{\infty}(y)\right)^{\frac{1}{1-p}}\right)^{p-1} \leq C \operatorname{card}(\mathcal{B})^{p} .
$$

Let us recall that $\mu^{\infty}\left(X_{j}^{n}\right)=H^{-n}$ for every $\boldsymbol{j} \in \mathfrak{I}^{n}$. Now, with $s=-\log _{\beta} H$ we obtain $\mu^{\infty}\left(X_{i}^{n}\right)=\beta^{s n}$. Notice also that from Hölder's inequality we have

$$
\begin{aligned}
\mu^{\infty}(E)^{p} & =\left(\int_{E} w(y)^{\frac{1}{p}} w(y)^{-\frac{1}{p}} d \mu^{\infty}(y)\right)^{p} \\
& \leq\left(\int_{E} w(y) d \mu^{\infty}(y)\right)\left(\int_{E} w(y)^{-\frac{1}{p-1}} d \mu^{\infty}(y)\right)^{p-1},
\end{aligned}
$$

for every $\mu^{\infty}$-measurable subset $E$ of $X$. Taking $E=X_{j}^{n}$ we obtain

$$
\left(f_{X_{j}^{n}} w(y) d \mu^{\infty}(y)\right)^{\frac{1}{1-p}} \leq f_{X_{j}^{n}} w(y)^{\frac{1}{1-p}} d \mu^{\infty}(y) .
$$

From the above inequality, the null overlapping, and Lemma 2.2 , we have that the left-hand side of 2.1 is bounded above by

$$
\begin{aligned}
& \left(\sum_{\boldsymbol{j} \in \mathcal{B}} f_{X_{j}^{n}} w(y) d \mu^{\infty}(y)\right)\left(\sum_{\boldsymbol{j} \in \mathcal{B}} f_{X_{j}^{n}} w(y)^{\frac{1}{1-p}} d \mu^{\infty}(y)\right)^{p-1} \\
& \quad \leq \frac{1}{C^{p} \beta^{n s p}}\left(\sum_{\boldsymbol{j} \in \mathcal{B}} \int_{X_{j}^{n}} w(y) d \mu^{\infty}(y)\right)\left(\sum_{\boldsymbol{j} \in \mathcal{B}} \int_{X_{j}^{n}} w(y)^{\frac{1}{1-p}} d \mu^{\infty}(y)\right)^{p-1} \\
& \leq \frac{1}{C^{p} \beta^{n s p}}\left(\int_{\bigcup_{j \in \mathcal{B}} X_{j}^{n}} w(y) d \mu^{\infty}(y)\right)\left(\int_{\bigcup_{j \in \mathcal{B}} X_{j}^{n}} w(y)^{\frac{1}{1-p}} d \mu^{\infty}(y)\right)^{p-1} \\
& \quad \leq \frac{1}{C^{p} \beta^{n s p}}\left(\int_{B\left(x, c_{1} r\right)} w(y) d \mu^{\infty}(y)\right)\left(\int_{B\left(x, c_{1} r\right)} w(y)^{\frac{1}{1-p}} d \mu^{\infty}(y)\right)^{p-1} \\
& \leq \frac{C_{p}}{C^{p} \beta^{n s p}} \mu^{\infty}\left(B\left(x, c_{1} r\right)\right)^{p}
\end{aligned}
$$


where $C_{p}$ denotes the Muckenhoupt constant for $w$ in $\left(X^{\infty}, \mu^{\infty}\right)$ and $x=\phi_{i}^{n}\left(x^{\infty}\right)$ for any $x^{\infty} \in X^{\infty}$ fixed. Notice that $x$ belongs to $X^{\infty}$, since $X^{\infty}=\bigcup_{i=1}^{H} \phi_{i}\left(X^{\infty}\right)$. Finally, we use the second inclusion in Lemma 2.2 to obtain

$$
\mu^{\infty}\left(B\left(x, c_{1} r\right)\right) \leq \sum_{\boldsymbol{i} \in \mathcal{B}\left(\boldsymbol{i}, c_{2} r\right)} \mu^{\infty}\left(B\left(\boldsymbol{\phi}_{\boldsymbol{j}}^{n}\left(x_{0}\right), \beta^{n}\right)\right) \leq C \beta^{n s} \operatorname{card}(\mathcal{B}),
$$

where in the last inequality we have used that $\left(X^{\infty}, d, \mu^{\infty}\right)$ is a normal space of dimension $s$ and item (2b) in Lemma 1.3

Hence, we have proved that $v(\boldsymbol{i}) \in A_{p}\left(\mathfrak{I}^{n}, \tilde{d}\right.$, card) with constant which does not depend on $n$, and consequently we have that $d \nu^{n}=w_{n} d \mu^{n}$ with $w_{n} \in A_{p}\left(X^{n}, d, \mu^{n}\right)$ uniformly in $n$.

Moreover, by definition of $\nu^{n}$ we have

$$
\begin{aligned}
\int_{X^{n}} f(x) w_{n}(x) d \mu^{n}(x) & =\sum_{i \in \mathfrak{I}^{n}} \int_{X_{i}^{n}} f(x) v(\boldsymbol{i}) d \mu_{\boldsymbol{i}}^{n}(x) \\
& =\sum_{\boldsymbol{i} \in \mathfrak{I}^{n}} H^{n} \int_{X^{n}} f(x) v(\boldsymbol{i}) \mathcal{X}_{X_{\boldsymbol{i}}^{n}}(x) d \mu^{n}(x) \\
& =\int_{X^{n}} f(x)\left(H^{n} \sum_{\boldsymbol{i} \in \mathfrak{I}^{n}} v(\boldsymbol{i}) \mathcal{X}_{X_{i}^{n}}(x)\right) d \mu^{n}(x) .
\end{aligned}
$$

Then

$$
w_{n}(x)=\sum_{\boldsymbol{i} \in \mathfrak{J}^{n}}\left(f_{X_{\boldsymbol{i}}^{n}} w(y) d \mu^{\infty}(y)\right) \mathcal{X}_{X_{i}^{n}}(x),
$$

where $\mathcal{X}_{A}$ denotes the indicator function of the set $A$.

Finally, we have to prove the weak star convergence. In order to do that, we shall fix a continuous function $\varphi$ on $X$. We shall prove that

$$
\lim _{n \rightarrow \infty} \int_{X} \varphi(x) w_{n}(x) d \mu^{n}(x)=\int_{X} \varphi(x) w(x) d \mu^{\infty}(x) .
$$

Notice first that

$$
\begin{aligned}
\int_{X} & \varphi(x) w_{n}(x) d \mu^{n}(x) \\
& =\int_{X} \varphi(x) \sum_{i \in \mathfrak{I}^{n}}\left(\frac{1}{\mu^{\infty}\left(X_{i}^{n}\right)} \int_{X} w(y) \mathcal{X}_{X_{i}^{n}}(y) d \mu^{\infty}(y)\right) \mathcal{X}_{X_{i}^{n}}(x) d \mu^{n}(x) \\
& =\sum_{i \in \mathfrak{I}^{n}} \int_{X} \int_{X} \varphi(x) \mathcal{X}_{X_{i}^{n}}(x) \frac{1}{\mu^{\infty}\left(X_{i}^{n}\right)} w(y) \mathcal{X}_{X_{i}^{n}}(y) d \mu^{\infty}(y) d \mu^{n}(x) \\
& =\int_{X} \sum_{i \in \mathfrak{I}^{n}}\left(\frac{1}{\mu^{\infty}\left(X_{i}^{n}\right)} \int_{X} \varphi(x) \mathcal{X}_{X_{i}^{n}}(x) d \mu^{n}(x)\right) \mathcal{X}_{X_{i}^{n}}(y) w(y) d \mu^{\infty}(y) \\
& =\int_{X} g_{n}(y) w(y) d \mu^{\infty}(y),
\end{aligned}
$$


where $g_{n}(y):=\sum_{i \in \mathfrak{I}^{n}}\left(\frac{1}{\mu^{\infty}\left(X_{i}^{n}\right)} \int_{X} \varphi(x) \mathcal{X}_{X_{i}^{n}}(x) d \mu^{n}(x)\right) \mathcal{X}_{X_{i}^{n}}(y)$. Therefore we have to show that

$$
\lim _{n \rightarrow \infty} \int_{X} g_{n}(y) w(y) d \mu^{\infty}(y)=\int_{X} \varphi(y) w(y) d \mu^{\infty}(y) .
$$

From the null overlapping property and the fact that $\mu^{\infty}\left(X_{i}^{n}\right)=\mu^{n}\left(X_{i}^{n}\right)=H^{-n}$, for each $y \in X^{\infty}$ we have

$$
\begin{aligned}
g_{n}(y) & =\frac{1}{\mu^{\infty}\left(X_{i_{0}}^{n}\right)} \int_{X} \varphi(x) \mathcal{X}_{X_{i_{0}}^{n}}(x) d \mu^{n}(x) \\
& =\frac{\mu^{n}\left(X_{i_{0}}^{n}\right)}{\mu^{\infty}\left(X_{i_{0}}^{n}\right)} f_{X_{i_{0}}^{n}} \varphi(x) d \mu^{n}(x) \\
& =f_{X_{i_{0}}^{n}} \varphi(x) d \mu^{n}(x),
\end{aligned}
$$

where $\boldsymbol{i}_{0}$ depends on $y$ and $n$. Since $X$ is compact, $\varphi$ is uniformly continuous on $X$, so that given $\varepsilon>0$ there exists $\delta>0$ such that $|\varphi(x)-\varphi(y)|<\varepsilon$ provided that $d(x, y)<\delta$. Let $N_{0}$ be such that $\beta^{n}<\delta$ if $n \geq N_{0}$. Hence, since $\operatorname{diam}\left(X_{\boldsymbol{i}_{0}}^{n}\right)=\beta^{n}$, for every $n \geq N_{0}$ we have

$$
\begin{aligned}
\left|g_{n}(y)-\varphi(y)\right| & =\left|f_{X_{i_{0}}^{n}}[\varphi(x)-\varphi(y)] d \mu^{n}(x)\right| \\
& \leq f_{X_{i_{0}}^{n}}|\varphi(x)-\varphi(y)| d \mu^{n}(x) \\
& <\varepsilon .
\end{aligned}
$$

Thus $\lim _{n \rightarrow \infty} g_{n}(y)=\varphi(y)$, and from the Lebesgue dominated convergence theorem we obtain

$$
\lim _{n \rightarrow \infty} \int_{X} g_{n}(y) w(y) d \mu^{\infty}(y)=\int_{X} \varphi(x) w(y) d \mu^{\infty}(y),
$$

and the theorem is proved.

\section{REFERENCES}

[1] H. Aimar and M. Carena, Pointwise estimate for the Hardy-Littlewood maximal operator on the orbits of contractive mappings, J. Math. Anal. Appl. 395 (2012), no. 2, 626-636. MR 2948254.

[2] H. Aimar, M. Carena and B. Iaffei, Completeness of Muckenhoupt classes, J. Math. Anal. Appl. 361 (2010), no. 2, 401-410. MR 2568704

[3] H. Aimar, M. Carena and B. Iaffei, Boundedness of the Hardy-Littlewood maximal operator along the orbits of contractive similitudes, J. Geom. Anal. 23 (2013), no. 4, 1832-1850. MR 3107681

[4] K. Falconer, Techniques in fractal geometry, John Wiley \& Sons, Ltd., Chichester, 1997. MR 1449135 
[5] J. García-Cuerva and J. L. Rubio de Francia, Weighted norm inequalities and related topics, North-Holland Mathematics Studies, 116, Notas de Matemática, 104, North-Holland, Amsterdam, 1985. MR 0807149

[6] J. E. Hutchinson, Fractals and self-similarity, Indiana Univ. Math. J. 30 (1981), no. 5, 713747. MR 0625600

[7] U. Mosco, Variational fractals, Ann. Scuola Norm. Sup. Pisa Cl. Sci. (4) 25 (1997), no. 3-4, 683-712. MR 1655537

[8] B. Muckenhoupt, Weighted norm inequalities for the Hardy maximal function, Trans. Amer. Math. Soc. 165 (1972), 207-226. MR 0293384

\section{Marilina Carena $a^{\bowtie}$}

Facultad de Ingeniería Química (UNL), researcher at CONICET, Departamento de Matemática (FIQ-UNL), Santa Fe, Argentina

marilcarena@gmail.com

Marisa Toschi

Instituto de Matemática Aplicada del Litoral (CONICET-UNL), Departamento de Matemática (FHUC-UNL), Santa Fe, Argentina

mtoschi@santafe-conicet.gov.ar

Received: September 23, 2018

Accepted: November 11, 2019 